\title{
Interpolation and approximation in decoding
}

\author{
Iwan Duursma
}

March 1998

\begin{abstract}
Recently Sudan formulated a decoding procedure for decoding RScodes beyond the packing radius. The potential of the method for AGcodes was recognized by Shokrollahi and Wasserman. We discuss similarities and differences with some previous algebraic decoding procedures.
\end{abstract}

\section{Introduction}

When Goppa introduced his famous construction of linear codes with algebraic curves over a finite field, he suggested that the decoding problem should be regarded as a problem of approximation of differentials. The various contributions to the solution of the decoding problem show that the actual algorithms are more easily formulated in terms of the dual code that is defined in terms of algebraic functions.

Other approaches emphasize interpolation as a way to solve the decoding problem. Thus the code itself may be defined in terms of algebraic functions and the decoding problem can be seen as looking for the best fit through a set of points in which some points are unreliable. The work by Sudan and by Shokrollahi and Wasserman shows the success of this approach in decoding beyond the packing radius of a code.

Characteristic for the approximation algorithms is the use of syndromes and the computation of unknown syndromes. These algorithms typically are fast for high rate codes with few errors. The interpolation method is fast for low rate codes. The decoding beyond the packing radius has so far only be achieved for low rate codes.

\section{A class of RS-codes}

The code $C$ of length $n$ and dimension $k$ over the field $F$ is said to be of type $[n, k, e, b]$ if any Hamming sphere of radius $e$ contains at most $b$ codewords [1].

For an analysis of the interpolation method we consider RS-codes with carefully chosen parameters. Let $X=X_{0} \cup \ldots \cup X_{b} \subset F$ be a partition of coordinates such that $X_{0}$ is of size $r$ and the remaining $b$ subsets are of size $m$. Let $f_{1}, \ldots, f_{b}$ be $b$ distinct polynomials of degree $r$ that are zero on $X_{0}$. The evaluation on $X$ 
of polynomials $f$ of degree at most $r$ defines a (punctured) RS-code of length $n=r+b m$ and dimension $k=r+1$. The word that is zero on $X_{0}$ and that has values $f_{j}(x)$ on $X_{j}$ is at distance $(b-1) m$ of each of the $b$ codewords $f_{j}(X)$.

Theorem: For given integers $b, r>0$, let $m=\left(\begin{array}{l}b \\ 2\end{array}\right)(r+1)$. let $C$ be a (punctured) RS-code of length $n=r+b m$ and dimension $k=r+1$. Any Hamming sphere of radius $e=(b-1) m$ contains at most $b$ codewords.

Proof: The codewords can be computed effectively by the interpolation method in [1].

Note that the codes have minimum distance $d=b m$ and that $e / d=(b-1) / b$.

\section{$3 \quad$ Factorization and syndromes}

The equations that are satisfied by the unknown syndromes are in general polynomial and are in general believed to be hard to solve (but see [2]). The interpolation method suggests that these equations can be solved efficiently (that is with the complexity of factorization) in a certain range of parameters. We therefore ask the following.

Problem: Does the vanishing ideal of the unknown syndromes have a special structure in the range of parameters where the interpolation method is successful?

We illustrate the problem for a code of type $[8,3,3,2](b=r=2$ in the theorem). For a received word $\left(y_{i}\right)$, the interpolation method first computes a polynomial

$$
\left(f_{0}\right) y^{2}+\left(g_{0}+g_{1} x+g_{2} x^{2}\right) y+\left(h_{0}+h_{1} x+h_{2} x^{2}+h_{3} x^{3}+h_{4}\right)
$$

that vanishes in the eight points $\left(x_{i}, y_{i}\right)$. Codewords at distance at most three from the received word $\left(y_{i}\right)$ are then obtained from the linear factors of the polynomial.

We assume that the coordinate set $X \subset F$ is the zero set of $x^{8}-x$ so that the code is extended cyclic. For known syndromes $S_{0}, S_{1}, S_{2}, S_{3}, S_{4}$, the unknown syndromes $S_{5}, S_{6}, S_{7}$ are such that the matrix

$$
\left(\begin{array}{llllllll}
S_{0} & S_{1} & S_{2} & S_{3} & S_{4} & S_{5} & S_{6} & S_{7} \\
S_{1} & S_{2} & S_{3} & S_{4} & S_{5} & S_{6} & S_{7} & S_{1} \\
S_{2} & S_{3} & S_{4} & S_{5} & S_{6} & S_{7} & S_{1} & S_{2} \\
S_{3} & S_{4} & S_{5} & S_{6} & S_{7} & S_{1} & S_{2} & S_{3}
\end{array}\right)
$$

has rank at most three. The full minors of the matrix define the vanishing ideal of the unknown syndromes. From the interpolation method we know that there are at most two syndrome extensions. Moreover they can be obtained efficiently.

We claim that the ideal contains the determinant of the matrix

$$
\left(\begin{array}{cccc}
S_{0} & S_{1} & S_{2} & S_{0}^{2}-2 S_{3} S_{4} \\
S_{1} & S_{2} & S_{3} & -S_{4}^{2} \\
S_{2} & S_{3} & S_{4} & -S_{1}^{2} \\
S_{3} & S_{4} & S_{5} & S_{5}^{2}-2 S_{1} S 2
\end{array}\right)
$$


Indeed, there are at most two possible solutions for the first unknown syndrome $S_{5}$.

\section{A previous result}

In [3], we give a fast erasure decoding scheme for low rate codes. It requires $3 k n$ field multiplications and is actually an implementation of the interpolation method for $b=1$.

\section{References}

[1] M. Sudan, "Decoding of Reed Solomon codes beyond the error correction bound," J. Complexity, vol. 13, no. 1, pp. 180-193, 1997.

[2] V.M. Sidelnikov, "Decoding the Reed-Solomon code when the number of errors is greater than $(d-1) / 2$, and zeros of polynomials in several variables." (Russian) Problemy Peredachi Informatsii, vol. 30, no. 1, pp. 51-69, 1994; (Translation) Problems Inform. Transmission, vol. 30, no. 1, pp. 44$59,1994$.

[3] I.M. Duursma, "On erasure decoding of AG-codes," Proceedings of ITW'94 (Moscow). Available at:

http://www.research.att.com/ duursma/pub. 